\title{
Information processing of olfactory stimuli by the dog: II. Stimulus control and sampling strategies in simultaneous discrimination learning
}

\author{
R. E. LUBOW \\ Tel-Aviv University, Ramat-Aviv, Israel \\ and \\ MOSHE KAHN and REUVEN FROMMER \\ Bar-Ilan University, Ramat Gan, Israel
}

\begin{abstract}
When two discrete stimuli are presented concurrently, with reinforcement contingent on a response to one of them $\left(S^{+}\right)$but not to the other $\left(S^{-}\right)$, the redundancy of the situation may allow discrimination by the organism on the basis of information provided by either stimulus or both stimuli. In addition, when the two stimuli are in different positions, only one stimulus need be sampled on any trial to determine where to respond. The actual locus of control ( $\mathrm{S}^{+}, \mathrm{S}_{-}^{-}$, or both) and the sampling strategy are not determinable within the standard acquisition paradigm. Answers to these problems were determined by introducing indifferent stimuli and nonstandard stimulus combinations to three dogs, each of which had previously acquired four simple olfactory discriminations. Results showed that each dog adopted an individual but highly consistent pattern of sampling and control.
\end{abstract}

In a two-choice discrimination paradigm, where, of each two stimuli presented, one is designated positive (i.e., a response in its presence is associated with reinforcement) and the other negative (i.e., a response in its presence is associated with the absence of reinforcement), it is unwarranted to assume that both stimuli serve equally to control responding. While it is possible that discriminative choice behavior is controlled by both stimuli, it would seem more parsimonious for the experimenter to assume, and for the organism to learn, that recognition of one member of the pair is sufficient to determine the correct choice. With positive control, the organism only needs to identify the location of the positive stimulus. If control is negative, once that stimulus has been identified and avoided, a response in the presence of any other stimulus will be reinforced.

The redundancy is maximal when both stimuli are presented simultaneously. If the first sampled stimulus is "positive" (or "not negative" for a negatively controlled organism), a response should be evoked immediately. If, on the other hand, it is "not positive" (or "negative"), a response should be evoked to the unsampled alternative. Since in such paradigms one stimulus position on each trial contains a positive stimulus, it is not necessary to sample the second stimulus to determine the site for a correct response.

A number of alternative paradigms solve these problems. In a go/no-go paradigm only one stimulus is presented on each trial, so the organism is forced to sample every presentation or risk error. Alternatively, more than two stimuli could be presented on each trial. While the number of samplings might still be limited to a maximum of $n-1$, more than one sampling would be necessary on any trial where the first sampled stimulus was not positive. Neither of these solutions deals with the locus of control problem.

The locus of control problem may be solved by making the discrimination relational. If the positive and negative stimuli vary freely along some stimulus dimension, maintaining only their relative positions on the scale, no discrimination is possible on the basis of one stimulus. By definition, once discrimination is acquired, locus of control has been established for the dimension and not for any specific stimuli. But there are many classes of discrete stimuli, particularly those operated on by the "lower senses," that are not readily placed on dimensional scales. Simultaneous presentation of discrete stimuli remains a convenient method for establishing such discriminations. A testing paradigm for determining locus of control and sampling strategy in these situations is presented. In such a test, in addition to $S^{+}$and $S-$, an associatively neutral stimulus $\left(\mathrm{S}^{0}\right)$ is introduced. The pair of stimuli on any trial is allowed to include any combination of stimuli, including two identical stimuli (double positives and double negatives). With this procedure the pattern of responding reveals the locus of control.

Table 1 describes four locus of control hypotheses and the pattern of results that would be predicted for each hypothesis when tested with three different stimulus pairs. Assume that the organism approached 
Table 1

Predicted Results for Different Locus of Control Hypotheses

\begin{tabular}{lccc}
\hline & \multicolumn{3}{c}{ Stimulus Pair } \\
\cline { 2 - 4 } Hypotheses & $\mathrm{S}^{+}$vs. $\mathrm{S}^{-}$ & $\mathrm{S}^{+}$vs. $\mathrm{S}^{0}$ & $\mathrm{~S}^{0}$ vs. $\mathrm{S}^{-}$ \\
\hline Null control & nd & nd & nd \\
Positive & $>$ & $>$ & nd \\
Negative & $>$ & nd & $>$ \\
Joint Control & $>$ & $>$ & $>$ \\
\hline
\end{tabular}

nd $=$ no difference

the first of two stimulus positions. Under the expanded paradigm, the stimulus may be positive, negative. or neufral. If the likelihood of responding to each of these stimuli is equal, the organism is not discriminating, i.e., is under null control.

Under all of the alternative possibilities of control, the probability of response to a positive stimulus is higher than that of a response to a negative stimulus. Therefore, this data, by itself, provides no information as to locus of control. However, when confronted with a neutral stimulus in this position, the probability of response is determined by the locus of control. If control is positive, the organism should avoid all nonpositive stimuli including the neutral one; the probability of a response to the neutral stimulus should be less than that to the positive and no different from that found to the negative (original nonpositive) stimulus, since both lack the locus of control equally.

On the other hand, an organism under negative control. should be equally likely to respond to either a positive or an indifferent stimulus more than to a negative stimulus, since both of the former are equally not negative. Only the subject under joint positive and negative control should show both a greater likelihood to respond to positives and a lesser likelihood to respond to negatives than to the undefined indifferent stimulus.

It is possible that within any given experiment some subjects are controlled by $S+$ and others by $\mathrm{S}-$. This hypothesis is given some plausibility by the fact that. when $S^{+}$is a compound, some subjects may come under control of one element of the compound, while others may come under control of a different element of the compound (Reynolds, 1961). Unfortunately, the various studies concerned with determining locus of control have failed to analyze the data from individual subjects.

The tests for locus of control which are described in Table 1 are also applicable to the case where the first sampled stimulus has been rejected and the second stimulus approached. By the definition of the situation, however, behavior toward the second stimulus is dependent on the first. While the first stimulus may be rejected for a variety of reasons (e.g., sampling errors may lead to rejection of a positive stimulus, or a positively controlled organism may reject a neutral stimulus in the first position), discussion will be limited to the case where the first sampled stimulus is negative.

In addition to predictions for positive, negative, or joint control, which are interpretable identically for responses in both positions, the null control comparisons are now meaningful; the organism which makes its discriminative decision after only one sampling will be equally likely to respond to any stimulus in the second position.

One further difference exists between behaviors in the two alternative positions. After rejecting a stimulus in the first presented position, the organism has an acquired alternative response, approaching the second position. But if the second stimulus is sampled and also rejected, no further response is readily available in its repertoire. While it may return to resample the first sampled stimulus, in cases where there is no "correct response" (i.e., double negative), the organism which has been well trained to make some response on every trial cannot be expected to demonstrate a zero response rate to the two alternatives. Thus, the proposed test is slightly loaded against the organism.

\section{METHOD}

\section{Subjects and Apparatus}

The subjects and apparatus were identical to those previously reported (Lubow, Kahn, \& Frommer, 1973). In that study, three mongrel dogs acquired four pairs of odor discriminations (Table 2 ). The test to be reported here occurred approximately $21 / 2$ weeks after the end of that study.

The apparatus consisted of two discrimination stations. $90 \mathrm{~cm}$ center to center. Each station was an open-front wooden box. $22 \times 22 \times 46 \mathrm{~cm}$, lined with Formica. A Lehigh-Valley omnidirectional manipulandum was vertically suspended from the top of the box, so that its lowest point was $12 \mathrm{~cm}$ from the box floor.

The stimuli odorous solutions were placed in open $250-\mathrm{ml}$ beakers beneath the manipulanda. Reinforcement, 190-mg Noyes dog-food pellets, was delivered from a Lehigh-Valley pellet dispenser. placed between the two discrimination stations. A $400-\mathrm{Hz}$ Sonalert tone source, which served as an end-of-trial signal, was located above the food dispenser.

\section{Procedure}

Baseline trials. Running procedure was similar to that previously reported. Each trial began with release of the dog from the start

Table 2

Odor Pairs Used in Training and Their Reinforcement Status for Each Dog

\begin{tabular}{|c|c|c|c|c|}
\hline \multirow[b]{2}{*}{ Pair } & \multirow[b]{2}{*}{ Stimulus } & \multicolumn{3}{|c|}{$\begin{array}{l}\text { Reinforcement Status } \\
\text { for Each Dog }\end{array}$} \\
\hline & & A & B & D \\
\hline \multirow{2}{*}{ W } & Acetone & + & + & + \\
\hline & Acetic Acid & - & - & - \\
\hline \multirow{2}{*}{$\mathbf{X}$} & Butanol & - & + & + \\
\hline & Ethanol & + & - & - \\
\hline \multirow{2}{*}{$\mathbf{Y}$} & 2-Propanol & - & + & - \\
\hline & Propylene-glycol & + & - & + \\
\hline \multirow{2}{*}{$\mathbf{Z}$} & Heptane & + & - & + \\
\hline & 1-Octanol & - & + & - \\
\hline
\end{tabular}


cage, which was $3 \mathrm{~m}$ in front of the feeder. Response latency was measured from the opening of the door until the first barpress response. In the event of a correct response, reinforcement was available for each response during a $12 \mathrm{sec}$ interval. A response to the negative stimulus terminated the trial immediately. Failure to respond within $10 \mathrm{sec}$ also terminated the trial. Termination of the trial was signaled by the tone, which continued until the dog reentered the start cage. Approximately $\mathbf{5 0}$ trials were run during each daily session. Only one stimulus pair was used in each session. The right-left position of the stimuli was determined randomly, with the limitation that the positive stimulus never appear more than twice successively in the same position.

Test trials. Test trials were run under conditions of extinction and terminated immediately after the first response. Each test trial was embedded in the baseline series, with from three to six baseline trials separating successive test trials. The test series, composed of six different trial types run in random order, consisted of trials with a combination of a positive and a negative, a positive and an indifferent, a negative and an indifferent, a double positive, a double negative, and a double indifferent stimulus. In all cases the indifferent stimulus was distilled water. Each stimulus combination appeared with each member equally in each stimulus position. The order of presentation of test trials was randomized in the same way as the baseline trials, with the additional provision that a test trial never occur after two baseline trials in which the positive stimulus appeared in the same position. Each stimulus combination under test appeared a total of 18 times for each odor stimulus pair. The number of appearances of each combination on each of the 15 test days was approximately equal.

\section{RESULTS AND DISCUSSION}

Analysis of the data was simplified by the strong tendency of all dogs to approach the left stimulus station first. This occurred on over $95 \%$ of the trials.

For analysis of the data, responses at each sampling station were cast into 2 by 2 tables, with one dimension being the stimuli under comparison and the second dimension "respond"/"did not respond." "Respond" signified a response to the approached position. "Did not respond" included both not responding to the sampled station, followed by a response to the alternative stimulus, and a termination of the $10-\mathrm{sec}$ response period without any response. Preliminary analysis of the responses showed no significant differences between response probabilities to each discriminative pair. There were, however, major differences between dogs. Analyses for overall performance are presented for each dog separately. In cases where all expected frequencies were greater than five, analysis was in the form of chi-square tests with one degree of freedom. The Yates correction was applied in all chi-square analyses. In the remaining cases, the Fisher exact probability test was used.

Table 3 presents data on the first approached stimulus for each dog. In reference to the predictions made in Table 1, it is clear that Dog $A$ is operating under positive control, responding to positive stimuli and rejecting all other stimuli. Dog D is clearly under negative control, responding equally readily to any nonnegative stimulus and avoiding only negatives. The case of Dog B is somewhat less clear. The nearly significant results of the $S^{0}$ greater than $S-$ probabilities suggests a tendency to be controlled by both positive and negative stimuli. Inspection of the data for the individual pairs indicates a higher negative control on the first two pairs than on the latter two. Positive control is high on all pairs.

The results for the second approached stimuli are presented in Table 4. No analysis was possible for data on the individual odor pairs because of the small sample sizes. Again, however, there did not appear to be any major differences between pairs, although in both Dogs A and B there is a tendency for control to be more pronounced on the first two pairs than on the latter two.

Dog A is under positive control, indicating two-stage positive control. That is, Dog A samples the odor at the first station and responds to it only if it is positive. If it is not positive, he samples the odor at the second station and responds to it only if it is positive.

Dog $\mathrm{D}$, however, shows no differential preference

Table 3

Responses to the First Approached Stimulus

\begin{tabular}{|c|c|c|c|c|c|c|c|c|}
\hline \multirow[b]{2}{*}{ Odor } & & \multicolumn{7}{|c|}{ Stimulus Pairs } \\
\hline & & \multicolumn{2}{|r|}{$\mathrm{S}^{+}$vs. $\mathrm{S}^{-}$} & \multicolumn{2}{|c|}{$\mathrm{S}^{+}$vs. $\mathrm{S}^{0}$} & \multicolumn{3}{|c|}{$\mathrm{S}^{0}$ vs. $\mathrm{S}^{-}$} \\
\hline A & $\begin{array}{l}\mathrm{F}^{*} \\
x^{2} \\
\mathrm{p}<\end{array}$ & $53 / 64$ & $\begin{array}{l}52.53 \\
.001\end{array}$ & $53 / 64$ & $\begin{array}{cc}42.95 & 15 / 64 \\
.001 & \end{array}$ & $15 / 64$ & $\begin{array}{l}.43 \\
\text { n.s. }\end{array}$ & $11 / 64$ \\
\hline B & $\begin{array}{l}\mathrm{F} \\
\chi^{2} \\
\mathrm{p} L\end{array}$ & $53 / 64$ & $\begin{array}{ll}47.58 & 13 / 64 \\
.001 & \end{array}$ & $53 / 64$ & $\begin{array}{l}27.07 \\
\quad .001\end{array}$ & $24 / 64$ & $\begin{array}{r}3.80 \\
.10\end{array}$ & $13 / 64$ \\
\hline D & $\begin{array}{l}\mathrm{F} \\
\chi^{2} \\
\mathrm{p} L\end{array}$ & $53 / 64$ & $\begin{array}{l}35.63 \\
.001 \\
\end{array}$ & $53 / 64$ & $\begin{array}{rl}3.19 & 44 / 65 \\
.10 & \end{array}$ & $44 / 65$ & $\begin{array}{l}16.57 \\
.001\end{array}$ & $19 / 63$ \\
\hline $\begin{array}{l}\text { All } \\
\text { Dogs }\end{array}$ & $\begin{array}{l}F \\
\chi^{2} \\
p<\end{array}$ & $159 / 192$ & $\begin{array}{c}173.40 \\
.001\end{array}$ & $159 / 192$ & $\begin{array}{l}63.62 \\
.001\end{array}$ & $83 / 193$ & $\begin{array}{c}17.36 \\
.001\end{array}$ & $43 / 191$ \\
\hline
\end{tabular}

*frequency expressed as ratio of responses to total approaches 
Table 4

Responses to Second Approached Stimulus when First Approached Stimulus was Negative

\begin{tabular}{|c|c|c|c|c|c|c|c|}
\hline \multirow[b]{2}{*}{ Odor } & & \multicolumn{6}{|c|}{ Stimulus Pairs } \\
\hline & & \multicolumn{2}{|c|}{$S^{+}$vs. $S^{-}$} & \multicolumn{2}{|c|}{$\mathrm{S}^{+}$vs. $\mathrm{S}^{0}$} & \multicolumn{2}{|c|}{$S^{0}$ vs. $S^{-}$} \\
\hline A & $\begin{array}{l}\mathrm{F}^{*} \\
\mathrm{x}^{2} \\
\mathrm{p} \angle\end{array}$ & $14 / 16$ & $8 / 32$ & $14 / 16$ & $7 / 16$ & $7 / 16$ & $8 / 32$ \\
\hline B & $\begin{array}{l}\mathrm{F} \\
\chi^{2} \\
\mathrm{p} \angle\end{array}$ & $14 / 16$ & $16 / 32$ & $14 / 16$ & $13 / 16$ & $13 / 16$ & $16 / 32$ \\
\hline D & $\begin{array}{l}\mathrm{F} \\
\chi^{2} \\
\mathrm{p} L\end{array}$ & $11 / 16$ & $20 / 32$ & $11 / 16$ & $12 / 16$ & $12 / 16$ & $20 / 32$ \\
\hline $\begin{array}{c}\text { All } \\
\text { Dogs }\end{array}$ & $\begin{array}{l}\mathrm{F} \\
\chi^{2} \\
\mathrm{p}<\end{array}$ & $39 / 48$ & $52 / 96$ & $39 / 48$ & $32 / 48$ & $32 / 48$ & $52 / 96$ \\
\hline
\end{tabular}

*frequency expressed as ratio of responses to total approaches

+ +exact probability for observed and all more extreme cases

for stimuli in the second position, being equally ready to respond to any stimulus. This indicates a one-stage sampling strategy, under control of the negative stimulus in the first sampled position. Dog D only samples the odor in the first position. If it is not negative, he responds to the odor at the second station, no matter what that odor is, i.e., without sampling it.

Dog B, again, presents an ambiguous picture in regard to control.

The data reported here are noteworthy on several counts. First of all, on a molar level of analysis, there was continued use of a position habit as part of a sampling strategy. Ali three subjects consistently first sampled one side of the apparatus, before either responding to that side or moving over to the second side. This is consistent with similar observations for rats (Hall, 1974; Mandler, 1966; Siegel, 1967) and pigeons (Honig, Beale, Seraganian, Lander, \& Muir, 1972). Furthermore, subjects in the present study exhibited this behavior even though they were under different modes of stimulus control. This suggests that the occurrence of a position habit is important for the development of more complex and successful strategies for the solution of a simultaneous discrimination problem. Rather than viewing a position habit, in terms of a persistent response to one side, as an erroneous hypothesis, it appears to be a building block for subsequent strategies. And, instead of being discarded in favor of other hypotheses, it is modified in form to become a permanent part of a sampling strategy.

Second, it appears that when data analysis is applied to individual subjects rather than to group data, it may be discovered that the type of stimulus control, $\mathrm{S}^{+}, \mathrm{S}-$, or both, is idiosyncratic. This is also true for sampling strategy.

Third, it will be recalled that Dog A exhibited positive stimulus control and a two-stage sampling strategy, while Dog D exhibited negative control and a one-stage sampling strategy. It remains to be determined whether there is a lawful relationship between type of control and sampling strategy.

Finally, these data raise several general questions. What is the distribution of the different types of stimulus control in different populations and under different conditions? And, most important, what are the antecedent conditions for the development of different stimulus control processes?

\section{REFERENCES}

HaLL, G. Response strategies after overtraining in the jumping stand. Animal Learning \& Behavior, 1973, 1, 157-160.

Hall, G. Strategies of simultaneous discrimination learning in the pigeon. Quarterly Journal of Experimental Psychology. 1974, 26. 520-529.

Honig, W. K., Beale, I., Seraganian, P., Lander, D., Muir, D. Stimulus and response reduction: Two aspeçts of inhibitory control in learning. In R. A. Boakes \& M. S. Halliday (Eds.), Inhibition and learning, London: Academic Press, 1972.

Lubow, R. E., KAHN, M., \& Frommer, R. Information processing of olfactory stimuli by the dog: I. The acquisition and retention of four odor-pair discriminations. Bulletin of the Psychonomic Society, 1973, 1, 143-145.

MAndler, J. M. Behavior changes during overtraining and their effects on reversal and transfer. Psychonomic Monograph Supplements, 1966, 1, 187-202.

Reynolds, G. S. Attention in the pigeon. Journal of the Experimental Analysis of Behavior, 1961, 4, 203-208.

SIEgEL, S. Overtraining and transfer processes. Journal of Comparative and Physiological Psychology, 1967, 64. 471-477.

Sutherland, N. S. Stimulus analyzing mechanisms. In Proceedings of a symposium on the mechanization of thought processes (Vol. 2). London: Her Majesty's Stationery Office, 1959. 